\title{
Eliciting dose is associated with tolerance development in peanut and cow's milk allergic children
}

\author{
C. Nitsche ${ }^{1,2,3^{*}}$ (D) C. D. Westerlaken-van Ginkel ${ }^{2,3}$, B. J. Kollen ${ }^{4}$, A. B. Sprikkelman²,3 G. H. Koppelman 2,3 \\ and A. E. J. Dubois ${ }^{2,3}$
}

\begin{abstract}
Background: Tolerance development rates differ between food allergies. Almost all previous studies have not used the gold standard method, the double-blind, placebo-controlled food challenge (DBPCFC), which may affect the reported prevalence rates. Little is known about the association of the eliciting dose (ED) obtained during the initial DBPCFC with later tolerance development.

Methods: This was a retrospective, tertiary care study of children who had a positive DBPCFC to either peanut, milk or egg, and at least one follow-up food challenge (open or DBPCFC) with the same food. The association between ED and negative (tolerant) follow-up food challenge outcome was analyzed by logistic regression, with adjustment for confounders. Suspected confounders were initial DBPCFC test characteristics, atopic comorbidities and serum specific lgE (slgE) levels.
\end{abstract}

Results: In 47 peanut allergic children, tolerance developed in 27.7\% (median follow-up duration of 43 months). In 80 milk (follow-up 23 months) and 55 egg (follow-up 37 months) allergic children, tolerance developed in 55.0\% and $65.5 \%$. The ED obtained during the initial DBPCFC was significantly associated with tolerance development in peanut and milk allergy, but not in egg allergy.

Conclusion: Approximately 1 out of 4 children with DBPCFC confirmed peanut allergy developed tolerance, compared to more than half of the children with milk or egg allergy, respectively. Tolerance development in peanut and milk allergy is significantly associated with ED at initial DBPCFC.

Keywords: Food allergy, Pediatrics, Eliciting dose, Prognosis, Atopic dermatitis

\section{To the Editor,}

Children allergic to cow's milk and hen's egg show a high rate of tolerance development over time $[1,2]$. Peanut allergy had been assumed to be a lifelong condition, until Hourihane et al. [3] reported a tolerance rate of $18 \%$ in peanut allergy.

*Correspondence: catharina.nitsche@gmail.com

${ }^{2}$ Department of Pediatric Pulmonology and Pediatric Allergology, University Medical Center Groningen, University of Groningen, CA43, PO BOX 30.001, 9700 RB Groningen, The Netherlands

Full list of author information is available at the end of the article
The gold standard diagnostic method, the doubleblind, placebo-controlled food challenge (DBPCFC) has not been consistently used to investigate the association of parameters available at diagnosis with tolerance development over time, particularly in peanut allergy.

Our aim was to analyze the association between the eliciting dose (ED) of the initial diagnostic DBPCFC with tolerance development and to identify confounding of this association by clinical parameters obtained during the initial DBPCFC.

Data was obtained from the database of the pediatric food challenge unit of the University Medical Center Groningen. Children allergic to peanut, cow's milk or 
hen's egg seen between 2001 and 2016 were included if they had a positive initial DBPCFC and at least one follow-up challenge (DBPCFC or open FC) with an interpretable outcome as part of routine clinical care. The only exclusion criterion for an initial DBPCFC was unwillingness to undergo the test, which was the case in $<2 \%$ of cases [4]. The outcome criteria and the procedure on the placebo and verum day including the dose scheme of the allergen doses given in steps were described elsewhere [5]. Complete avoidance of all forms of the tested food was advised. Follow-up was scheduled individually and the second food challenge was offered at least 1 year after diagnosis. An open FC was chosen for follow-up, if there was no evidence of tolerance or persistent food allergy after diagnosis such as uneventful unequivocal ingestion or allergic reaction to the food. We defined tolerance as a negative challenge (DBPCFC or open FC) after an initial positive DBPCFC. Persistent allergy was defined as positivity of all challenge test results during the follow-up period.

The following factors were analyzed: gender; age; coexistent eczema, asthma, allergic rhinitis, number of comorbid food allergies and the most severe previous accidental reaction (hereafter referred to as index reaction). In addition, the following parameters pertaining to the initial DBPCFC were analyzed: ED, severity of the challenge reaction and allergen specific Immunoglobulin $\mathrm{E}$ (sIgE) level. To determine the severity of the reaction during the initial DBPCFC and of the index reaction, we used a previously described scoring system. Symptoms of the most severe reaction during the FC, recorded by trained allergy nurses, were scored by body part (see Table 1) and summed to compute a severity index [6]. The levels of sIgE for each allergen were measured using the Pharmacia CAP System (Thermo Fisher Scientific, Uppsala, Sweden).

Logistic regression analyses were performed to estimate the association between clinical factors known at the time of the initial DBPCFC and tolerance development for each food group separately. The association between ED and tolerance development was adjusted for

Table 1 Scoring system of reaction severity by van der Zee et al. [6]

\begin{tabular}{ll}
\hline Body part & $\begin{array}{l}\text { Severity } \\
\text { score }\end{array}$ \\
\hline Skin & 1 \\
Gastrointestinal & 2 \\
Upper airway (nose, eyes, throat) & 3 \\
Lower airway (lungs) & 3 \\
Cardiovascular or neurological & 3 \\
\hline
\end{tabular}

confounding by clinical factors for each food separately. A factor changing the beta coefficient of the relationship between the ED and tolerance by $10 \%$ or more was defined as confounder. The minimum number of children in the smallest group (persistently allergic versus tolerant group) generating sufficient events per variable (EPV) in a model with sufficient power was set at five [7] (i.e. meeting the EPV rule). In order to comply with the EPV rule we were unable to test all potential factors for confounding in one model. We chose to fit the strongest confounder in the model for adjustment based on the largest deviation from the unadjusted beta coefficient. To take the lower specificity of the open FC into account, we calculated tolerance rates in the food groups including only children followed-up by DBPCFC in a separate sensitivity analysis. The Kaplan-Meier survival curve was only used to visualize the course of survival of tolerance development of the three foods over time. No log rank test was used to directly compare the individual courses. All statistical tests were performed two sided at $<0.05$. IBM ${ }^{\circledR}$ SPSS ${ }^{\circledR}$ Statistics v23, was used for all analyses.

Of 1332 children who initially underwent a DBPCFC, 47 children allergic to peanut, 80 children allergic to cow's milk and 55 allergic to hen's egg met the inclusion criteria (see Fig. 1).

Tolerance was diagnosed in $27.7 \%(13 / 47)$ of children allergic to peanut after a median follow-up duration of 43 months, in $55.0 \%$ (44/80) and in 65.5\% (36/55) of children allergic to cow's milk and hen's egg after 23 and 37 months respectively (see Table 2). Eliciting dose was significantly associated with tolerance development $(\mathrm{OR}=1.004,95 \%$ CI $1.001-1.007, \mathrm{p}=0.010$ for peanut; $\mathrm{OR}=1.001,95 \% \mathrm{CI} 1.000-1.001, \mathrm{p}=0.001$ for cow's milk; $\mathrm{OR}=1.001,95 \% \mathrm{CI} 1.000-1.001, \mathrm{p}=0.038$ for hen's egg; unadjusted). Positive history of eczema was identified as a confounder of the association between ED and tolerance development in peanut allergic children $(\mathrm{OR}=1.005,95 \%$ CI $1.001-1.008, \mathrm{p}=0.009$, adjusted for eczema). No confounder was identified for the reported association for cow's milk. In hen's egg allergic children, the association did not reach significance after adjustment $(\mathrm{OR}=1.000$, $95 \%$ CI 1.000-1.001, $\mathrm{p}=0.299$, adjusted).

A double-blind instead of an open follow-up challenge was performed in 24 of 47 (51.1\%) peanut, in 28 of 80 (35.0\%) cow's milk and in 17 of 55 (30.9\%) hen's egg allergic children. Considering only cases followed up by DBPCFC, as many as $33.3 \%$ peanut allergic children developed tolerance. Similarly, in $75.0 \%$ of milk allergic and $76.5 \%$ of hen's egg allergic children developed tolerance, which is higher than rates observed in previous studies $[1,2]$.

This is the first study in peanut allergic children using exclusively the DBPCFC for diagnosis at baseline to 


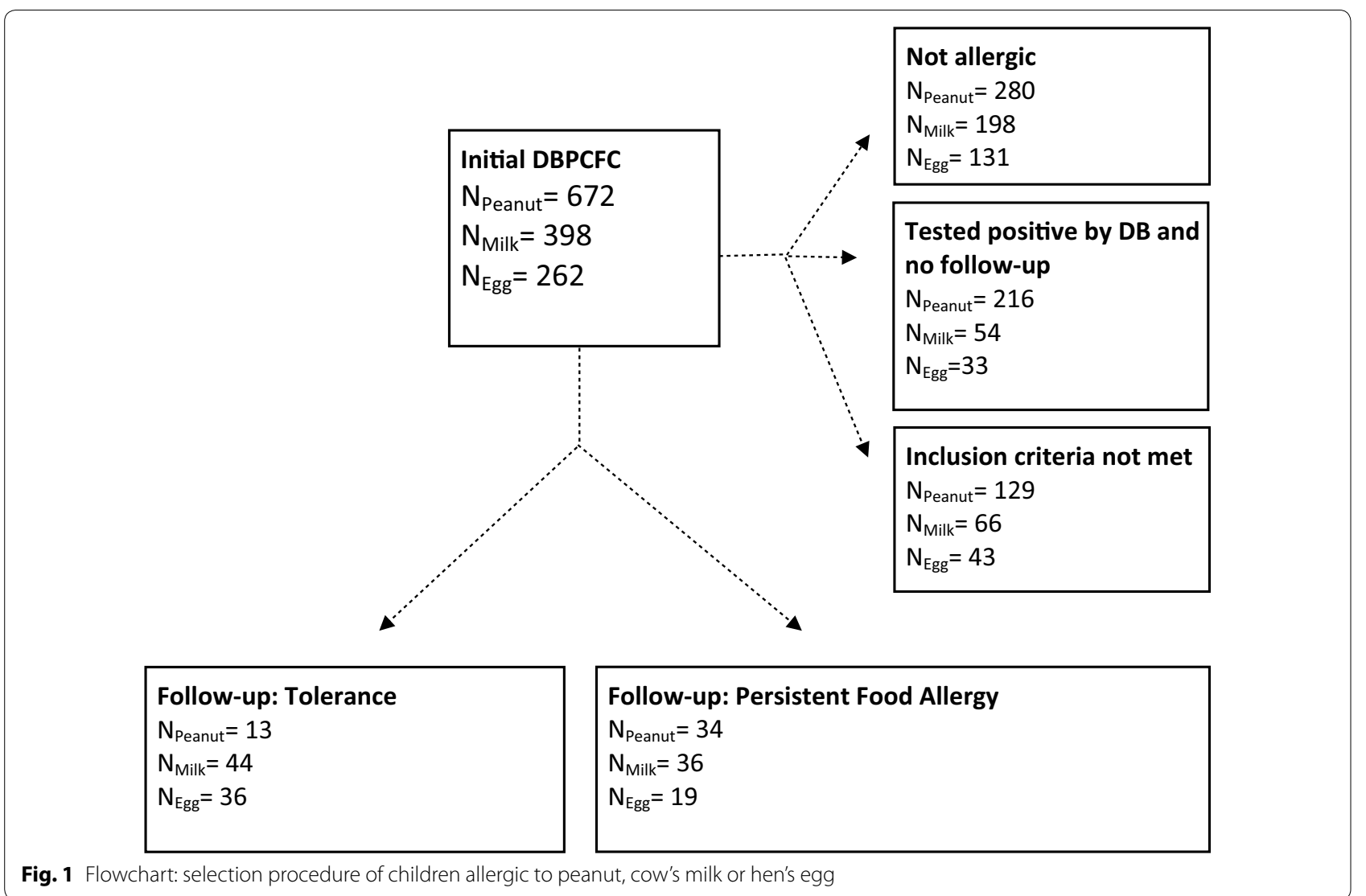

investigate the association between the ED at diagnosis and tolerance development. False-positive rates up to $70 \%$ reported for open FCs $[8,9]$ may lead to initial overestimation of food allergy and to subsequent underestimation of tolerance development.

Comparing the three food groups, a high eliciting dose was a common clinical parameter associated with tolerance in peanut and cow's milk, but not in hen's egg allergy when adjusted for confounding.

Despite the fact that this study was carried out using a large database, the power of analysis was still limited, leading to a limited ability to test for multiple confounders in the same association model. Although the data base includes essential patient information such as comorbid atopic diseases, it is difficult to compare these results with the general population or primary care since this research describes data of children who were referred to a tertiary care clinic with suspected food allergy. In 216 peanut, in 54 cow's milk and in 33 hen's egg allergic children, the outcome of only one DBPCFC was available and no follow up tests had yet been done (see Fig. 1). This was the case when the initial diagnosis was recent, if there had been clear reactions to accidental exposure during the follow up period or in rare cases, if the food has been reintroduced into the diet without medical approval. Figure 2 presents a visualization of the course of survival of tolerance development in each group over the duration of follow-up (in months). The declining curves represent the increasing number of subjects who developed tolerance over time.

In summary, our data shows that tolerance development is common for peanut allergy as well as for cow's milk and hen's egg allergy, and that ED is associated with tolerance development in peanut and cow's milk allergy. Factors associated with persistent food allergy may support clinicians to give prognosis but still cannot replace current diagnostic tools with the DBPCFC being the preferred test. 


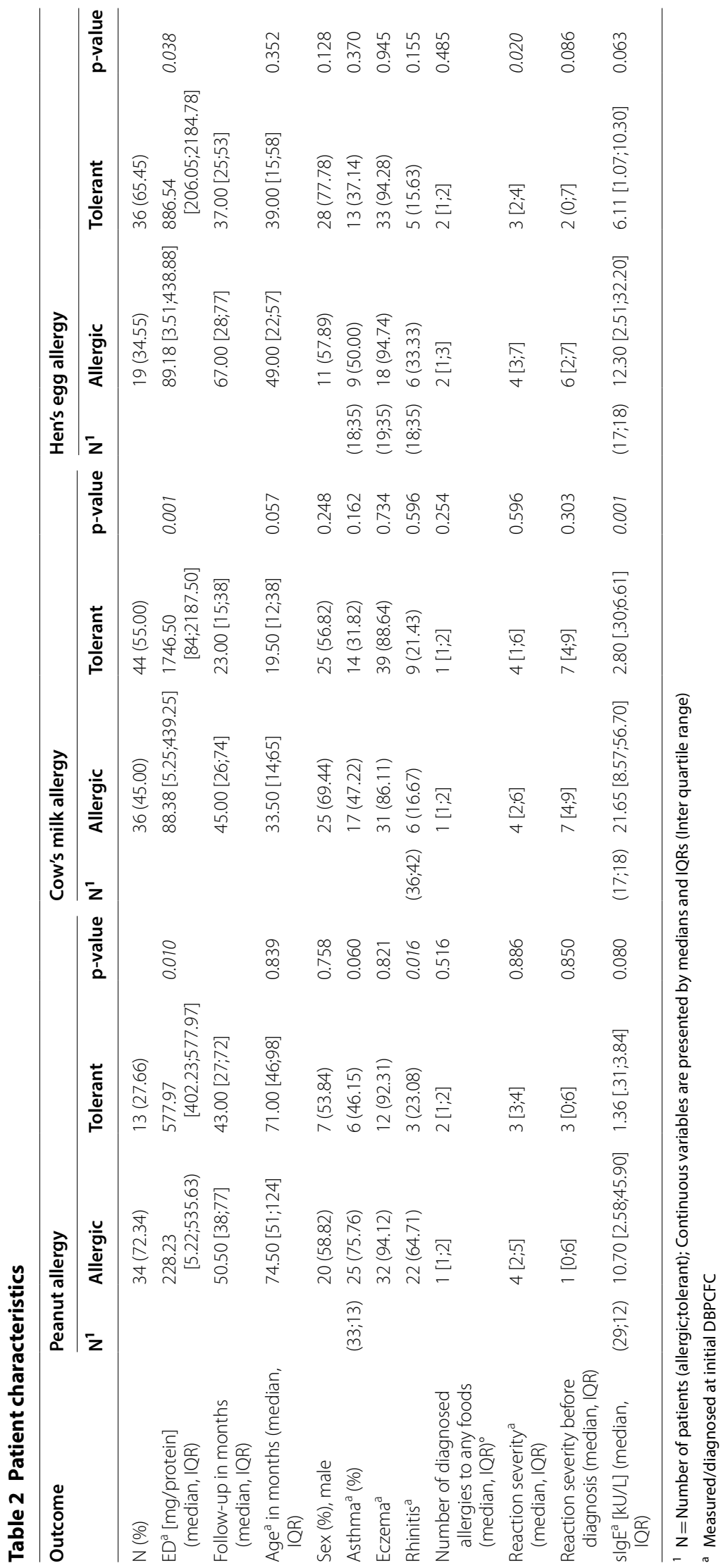




\section{Tolerance Development}

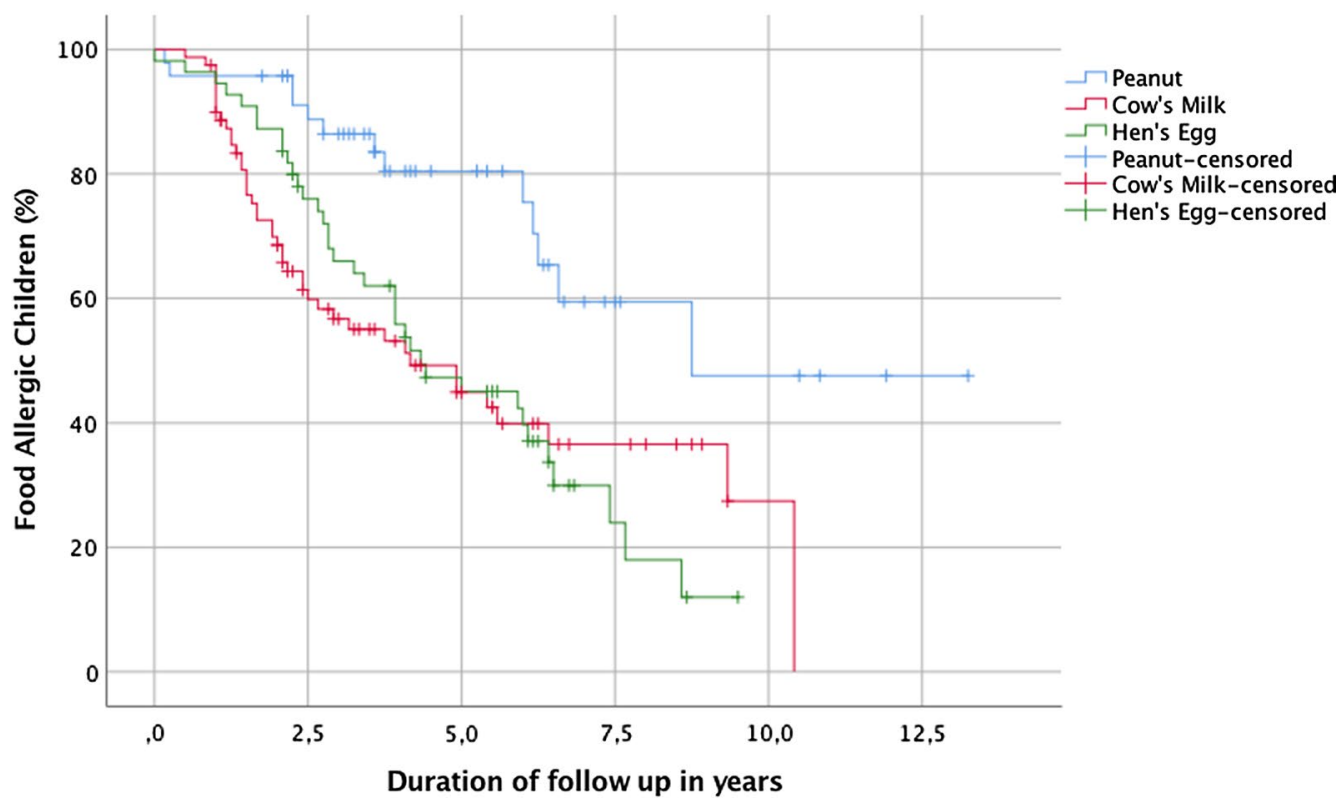

Fig. 2 Kaplan-Meier survival curve in peanut, cow's milk and hen's egg allergic children

\section{Abbreviations}

DBPCFC: double-blind, placebo-controlled food challenge; FC: food challenge; ED: eliciting dose; slgE: specific immunoglobulin E.

\section{Acknowledgements}

Not applicable.

\section{Authors' contributions}

Conception of the idea for the study (CN, CDVG, AEJD). Expert advice and supervision (AEJD). Data analysis and preparation of manuscript (CN). Actively contributed to writing, editing and evaluation of the manuscript (CN, CDVG, BJK, ABS, GHK, AEJD). All authors read and approved the final manuscript.

\section{Funding}

This research received no specific Grant from any funding agency in the public, commercial, or not-for-profit sectors.

\section{Availability of data and materials}

The dataset analysed during the current study is not publicly available due to institutional use only but is available from the corresponding author on reasonable request.

\section{Ethics approval and consent to participate}

This study was deemed exempt from medical ethical approval by the University Medical Center Groningen institutional Medical Ethical Committee.

\section{Consent for publication}

Not applicable.

\section{Competing interests}

The authors declare, that they have no relevant conflict of interest regarding this manuscript. The authors report to have received funding from the Nutricia Research Foundation, GSK, TEVA the Netherlands, UBBO EMMIUS Foundation, TETRI Foundation, Lung Foundation of the Netherlands, which had no relation to the submitted work.

\section{Author details}

${ }^{1}$ University of Oldenburg, Oldenburg, Germany. ${ }^{2}$ Department of Pediatric Pulmonology and Pediatric Allergology, University Medical Center Groningen, University of Groningen, CA43, PO BOX 30.001, 9700 RB Groningen, The Netherlands. ${ }^{3}$ GRIAC Research Institute, University Medical Center Groningen, University of Groningen, Groningen, The Netherlands. ${ }^{4}$ Department of General Practice and Elderly Care Medicine, University Medical Center Groningen, University of Groningen, Groningen, The Netherlands.

Received: 6 May 2019 Accepted: 23 October 2019

Published online: 06 November 2019

\section{References}

1. Schoemaker AA, Sprikkelman AB, Grimshaw KE, et al. Incidence and natural history of challenge-proven cow's milk allergy in European childrenEuroPrevall birth cohort. Allergy. 2015;70(8):963-72.

2. Peters RL, Dharmage SC, Gurrin LC, et al. The natural history and clinical predictors of egg allergy in the first 2 years of life: a prospective, population-based cohort study. J Allergy Clin Immunol. 2014;133(2):485-91.

3. Hourihane JO, Roberts SA, Warner JO. Resolution of peanut allergy: casecontrol study. BMJ. 1998;316(7140):1271-5.

4. Blom WM, Vlieg-Boerstra BJ, Kruizinga AG, van der Heide S, Houben GF, Dubois AE. Threshold dose distributions for 5 major allergenic foods in children. J Allergy Clin Immunol. 2013;131(1):172-9.

5. Pettersson ME, Koppelman GH, Flokstra-de Blok BMJ, Kollen BJ, Dubois AEJ. Prediction of the severity of allergic reactions to foods. Allergy. 2018;73:1532-40. https://doi.org/10.1111/all.13423

6. van der Zee T, Dubois A, Kerkhof M, van der Heide S, Vlieg-Boerstra B. The eliciting dose of peanut in double-blind, placebo-controlled food challenges decreases with increasing age and specific lgE level in children and young adults. J Allergy Clin Immunol. 2011;128(5):1031-6.

7. Vittinghoff $E$, McCulloch CE. Relaxing the rule of ten events per variable in logistic and Cox regression. Am J Epidemiol. 2007;165(6):710-8. 
8. Pastorello EA, Pravettoni V, Stocchi L, Bigi A, Schilke ML, Zanussi C. Are double-blind food challenges necessary before starting an elimination diet? Allergy Proc. 1991;12(5):319-25.

9. Venter $C$, Pereira B, Voigt $K$, et al. Comparison of open and double-blind placebo-controlled food challenges in diagnosis of food hypersensitivity amongst children. J Hum Nutr Diet. 2007;20(6):565-79.

\section{Publisher's Note}

Springer Nature remains neutral with regard to jurisdictional claims in published maps and institutional affiliations.
Ready to submit your research? Choose BMC and benefit from:

- fast, convenient online submission

- thorough peer review by experienced researchers in your field

- rapid publication on acceptance

- support for research data, including large and complex data types

- gold Open Access which fosters wider collaboration and increased citations

- maximum visibility for your research: over $100 \mathrm{M}$ website views per year

At BMC, research is always in progress.

Learn more biomedcentral.com/submissions 